\title{
Material identification with a filter array-detector array infrared microspectrometer: numerical study
}

\author{
Jiajun Meng $^{1 *}$, Jasper J. Cadusch ${ }^{1}$, Kenneth B. Crozier ${ }^{1,2^{*}}$ \\ ${ }^{1}$ Department of Electrical and Electronic Engineering, University of Melbourne, Victoria 3010, Australia \\ 2 School of Physics, University of Melbourne, Victoria 3010, Australia \\ *Authore-mail address: jiajunm@student.unimelb.edu.au,kenneth.crozier@unimelb.edu.au
}

\begin{abstract}
We design a plasmonic filter array for a filter array-detector array microspectrometer. We perform numerical experiments, including noise, that predict that this configuration would enable the identification of various materials via their infrared fingerprints. (C) 2020 The Author(s) OCIS codes: $040.6808250 .5403 ; 280.4788$
\end{abstract}

\section{Introduction}

Numerous applications exist for the identification of materials via their mid-infrared fingerprints. These include biosensing, industrial control and environmental monitoring. At the time of writing, the workhorse tool for midinfrared spectroscopy is the Fourier transform infrared (FTIR) spectrometer. Such tools are relatively large, heavy and costly. For many applications, this is not problematic. However, there is currently a move toward miniaturized optical systems, to enable their incorporation into consumer electronic devices such as smartphones. This motivates the development of small, light and inexpensive mid-infrared spectrometers. That is the topic of this study.

Here, we perform a numerical study on a system that comprises a filter array detector array (FADA) spectrometer. The filter array is designed to cover the mid- to long-wave infrared and comprises a plasmonic metasurface. It has been recently shown that metasurfaces present exciting opportunities for molecular sensing $[1,2]$. Our model assumes a detector array consisting of an uncooled thermal camera of a design whose footprint is comparable to that of a coin. We perform numerical experiments that demonstrate material identification by readout of the detector array.

\section{Device Design}

a).

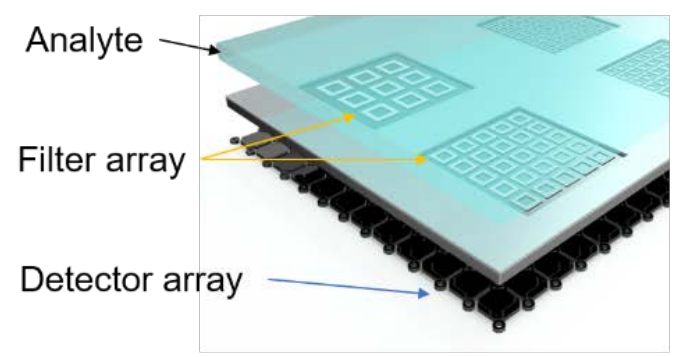

b).

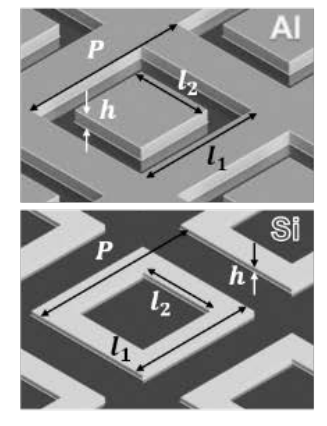

c).

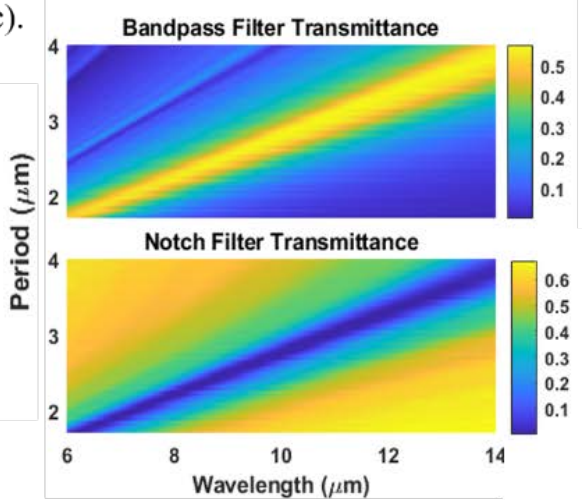

Figure 1: (a) Device schematic (b) Top: rectangular ring aperture on aluminum film. Bottom: rectangular aluminum ring (c) Simulated bandpass and notch filter transmittance spectra vs periods. Period sweeps from $1.7 \mu m$ to $4 \mu m$.

The schematic of the FADA spectrometer we propose is depicted in Fig. 1a. This platform would integrate a plasmonic filter array with a detector array. Light from an infrared lamp or LED (not shown in Fig, 1a) would illuminate the analyte, with the transmitted signal impinging upon the FADA spectrometer. As we demonstrate in this study, readout of the detector array will enable identification of the analyte. The accuracy of this detection will of course depend on detector noise. We therefore include the latter in our model. It also depends on the responsivity of the detector array. We therefore take the responsivity spectrum as being that of an uncooled detector array that, at the time of writing, has been incorporated into consumer electronics, the FLIR Tau Lepton. This uses a microbolometer array and covers the wavelength range 6 to $14 \mu \mathrm{m}$. We furthermore assume that our filter array contains only 20 filters, to ensure that it would be cost effective for manufacturing and for integration with the detector array. 
Undoped silicon is chosen as the substrate for the plasmonic filter arrays, due to its widespread use in nanofabrication. It furthermore exhibits a relatively high transmittance in the mid- to long-wave IR range (6 to 14 $\mu \mathrm{m}$ ) of $\sim 50 \%$ for a standard wafer thickness of $500 \mu \mathrm{m}$. Aluminum is chosen as the plasmonic material, due to its low cost. The filters are divided comprise two categories: notch filters (square arrays of rectangular rings) and bandpass filters (square arrays of rectangular coaxial apertures). Unit cells of the two filter types are shown in Fig. $1 \mathrm{~b}$. The geometric parameters $l_{1}, l_{2}, P, h$ (Fig. $1 \mathrm{~b}$ ) govern the resonances supported by the plasmonic structures. By appropriate choice of these parameters, therefore, the location of notches and bandpass features can be tuned. We have previously used related structures for computational IR spectroscopy [3, 4]. Finite difference time domain (FDTD) simulations of the filters are presented as Fig. 1c. These show transmission spectra as a function of array period $P$, with the other geometric parameters given as follows. For the notch filters we have: $\left\{l_{1}, l_{2}, h\right\}=$ $\{0.5 P, 0.3 P, 50 \mathrm{~nm}\}$. For the bandpass filters, we have $\left\{l_{1}, l_{2}, h\right\}=\{0.6 P, 0.4 P, 140 \mathrm{~nm}\}$. It can be seen that the transmission notches and bandpass features both shift to longer wavelengths with the period. Typical FWHM of bandpass filters is $3.1 \mu \mathrm{m}$ while is the typical FWHM of notch filters is $2.2 \mu \mathrm{m}$.

\section{Result and Discussion}

The output of each FADA element can be modeled as, $O_{f_{k}}=n_{k}+\int_{\lambda_{1}}^{\lambda_{2}} I \cdot T_{f_{k}} \cdot T_{M} \cdot R_{F L I R}$, where $I$ is the intensity spectrum of the infrared light source, $T_{M}$ is the analyte transmittance, $R_{F L I R}$ is the responsivity of the Lepton camera module, $O_{f_{k}}, n_{k}, T_{f_{k}}$ are the output, total noise and transmittance of $k^{t h}$ filter element, respectively. The output is normalized using a reference output in which there is no analyte in the optical path length presented. The impact of noise can be reduced by introducing frame averaging to achieve a higher overall signal to noise ratio (SNR). In the numerical experiment, we employ Gaussian noise with SNR of 200. The responsivity spectrum is taken from the datasheet of the detector array [5]. We choose to average 81 frames. The normalized value $\tilde{O}_{f_{k}}$ after frame averaging can be calculated using $\tilde{O}_{f_{k}}=E\left[O_{f_{k}}\right] / E\left[e_{k}+\int_{\lambda_{1}}^{\lambda_{2}} I \cdot T_{f_{k}} \cdot R_{F L I R}\right]$, where $e_{k}$ is the noise of the reference output.

To test our approach, we use nine analyte spectra, downloaded from the NIST Chemistry WebBook. The transmission spectra of these analytes are shown as Fig 2a. For the liquids, the path length is assumed to be $0.01 \mathrm{~cm}$. Ethanol, isopropanol, and acetone are assumed to have concentrations of $10 \%$ (in carbon tetrachloride). For the gases, we assume $25 \mathrm{mmHg}$ torr of gas is diluted in nitrogen to $600 \mathrm{mmHg}$, with an optical path length of $10 \mathrm{~cm}$. The IR light source is taken as blackbody radiation at $373.15 \mathrm{~K}$. The normalized outputs of different materials are shown in Fig. 2b and Fig. 2c. It can be seen that the different materials result in highly distinctive output signals. We conclude that the system would be able to distinguish between the materials studied.

a).
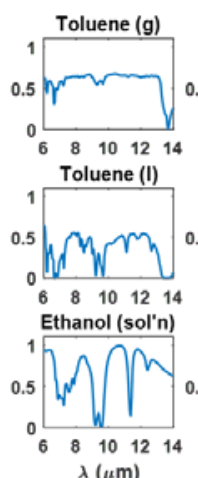

Material Transmittance
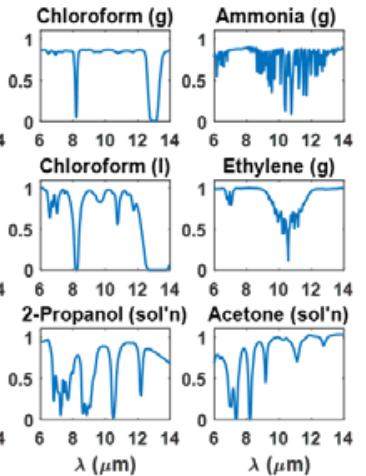

b). Bandpass Filter Normalized Output

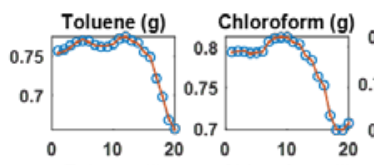

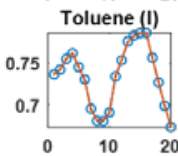
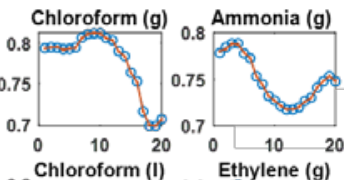

c). Notch Filter Normalized Output
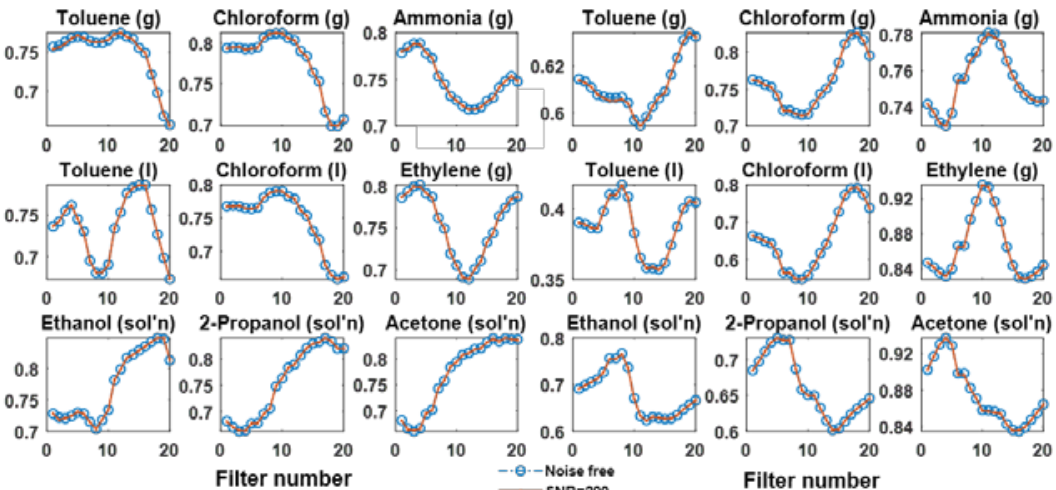

Figure 2: (a) Transmittance spectra of nine materials (b) Bandpass filter normalized output (c) Notch filter normalized output

\section{References}

[1] A. Tittl, A. Leitis, M. Liu, F. Yesilkoy, D.-Y. Choi, D. N. Neshev, Y. S. Kivshar, and H. Altug, "Imaging-based molecular barcoding with pixelated dielectric metasurfaces,” Science 360, 1105-1109 (2018).

[2] A. Leitis, A. Tittl, M. Liu, B. H. Lee, M. B. Gu, Y. S. Kivshar, and H. Altug, "Angle-multiplexed all-dielectric metasurfaces for broadband molecular fingerprint retrieval," Sci Adv 5, eaaw2871 (2019).

[3] B. J. Craig, J. Meng, V. R. Shrestha, J. J. Cadusch, and K. B. Crozier, "Mid- to long-wave infrared computational spectroscopy using a subwavelength coaxial aperture array,” Sci Rep 9, 13537 (2019).

[4] B. Craig, V. R. Shrestha, J. Meng, J. J. Cadusch, and K. B. Crozier, "Experimental demonstration of infrared spectral reconstruction using plasmonic metasurfaces,” Opt Lett 43, 4481-4484, (2018).

[5] FLIR, FLIR LEPTON Engineering Datasheet, (2019). 


\section{University Library}

\section{- M M I N E R VA A gateway to Melbourne's research publications}

Minerva Access is the Institutional Repository of The University of Melbourne

Author/s:

Meng, J;Cadusch, JJ;Crozier, KB

Title:

Material identification with a filter array-detector array infrared microspectrometer: Numerical study

Date:

2020-09-14

Citation:

Meng, J., Cadusch, J. J. \& Crozier, K. B. (2020). Material identification with a filter arraydetector array infrared microspectrometer: Numerical study. Optics InfoBase Conference Papers, pp.ftu2e.6-. Optica Publishing Group. https://doi.org/10.1364/FIO.2020.FTu2E.6.

Persistent Link:

http://hdl.handle.net/11343/294866 